\title{
Estabilidad de Taludes del Tajo Abierto Jésica considerando el Macizo Rocoso Isotrópico y Anisotrópico
}

\author{
Slope Stability of the Open Pit Jésica considering the Isotropic and \\ Anisotropic Rock Mass
}

Editta Herrera Carranza ${ }^{1}$, Lloyd Williams Solórzano Poma ${ }^{2}$

Recibido: 01/07/2020 - Aprobado: 30/10/2020 - Publicado: 30/11/2020

\begin{abstract}
RESUMEN
El trabajo de investigación analiza la estabilidad del talud global, en seis (6) tipos de alteración hidrotermal presentes en el tajo abierto Jésica, considerando al macizo rocoso isotrópico es decir que no tiene orientación preferencial de discontinuidades y anisotrópico teniendo en cuenta las discontinuidades paralelas o subparalelas al talud. Para el análisis de estabilidad de taludes del macizo rocoso isotrópico y anisotrópico se utilizó el criterio de rotura de Hoek-Brown Generalizado y Anisotrópico Generalizado respectivamente, ambos en el software Slide versión 6.0.

En el análisis de estabilidad anisotrópico se consideraron dos (2) familias de discontinuidades subparalelas al talud F1 (buzamiento de $72^{\circ}$ a $82^{\circ}$ ) y F2 (buzamiento de $45^{\circ}$ a $55^{\circ}$ ). Los factores de seguridad obtenidos considerando el macizo rocoso anisotrópico son menores hasta en $29 \%$ respecto a los obtenidos en el macizo rocoso isotrópico; así mismo a mayor calidad del macizo rocoso, mayor es la variación de los factores de seguridad, esta diferencia es más marcada en la zona de sulfuros con la familia F2, donde en un macizo rocoso con RMR 40 el factor de seguridad disminuye en $8 \%$, mientras que en un macizo rocoso con RMR 61 el factor de seguridad disminuye en $29 \%$.
\end{abstract}

Palabras claves: Alteración hidrotermal; anisotrópico; estabilidad de taludes; isotrópico; macizo rocoso.

\begin{abstract}
The research paper analyzes the stability of the global slope, in six types of hydrothermal alteration present in the open pit Jésica, identifies the isotropic rock mass, that is, it does not have preferential orientation of discontinuities and anisotropic taking into account the parallel or sub-parallel discontinuities to the slope. For the slope stability analysis of the isotropic and anisotropic rock mass, the Generalized Hoek-Brown and Generalized Anisotropic failure criteria were used respectively, both in Slide 6.0 software.

Two sets of discontinuities sub-parallel to the slope were considered in the anisotropic stability analysis F1 (dip from $72^{\circ}$ to $82^{\circ}$ ) y $\mathrm{F} 2$ (dip from $45^{\circ}$ to $55^{\circ}$ ). The safety factors obtained considering the anisotropic rock mass are up to $29 \%$ lower than those obtained in the isotropic rock mass; as well as the higher quality of the rock mass, the greater the variation of the safety factors, this difference is more marked in the sulphides zone with the set F2, where in a rock mass with RMR 40 the safety factor decreases by $8 \%$, while in a rock mass with RMR 61 the safety factor decreases by $29 \%$.
\end{abstract}

Keywords: Hydrothermal alteration; anisotropic; slope stability; isotropic; rock mass.

\footnotetext{
1 Universidad Nacional Mayor de San Marcos, Facultad de Ingeniería Geológica, Minera, Metalúrgica y Geográfica, Posgrado en Ingeniería Geológica. Lima, Perú. Autor para correspondencia: editta.herrera@gmail.com ORCID: $h$ htps://orcid.org/0000-0003-1147-428X

2 Docente de la Universidad Nacional Mayor de San Marcos, Facultad de Ingeniería Geológica, Minera, Metalúrgica y Geográfica, Posgrado en Ingeniería Geológica. Lima, Perú. E-mail: Isppoma@yahoo.com
} 


\section{INTRODUCCIÓN}

El tema en particular consistió en realizar el análisis de estabilidad de taludes considerando el macizo rocoso isotrópico (falla circular) y anisotrópico (falla no circular), para los cuales se utilizó el método Morgenstern-Price y los criterios de rotura de Hoek-Brown Generalizado y Anisotrópico Generalizado en el software Slide versión 6.0 del programa Rocscience Inc.

El criterio de rotura de Hoek- Brown Generalizado, es un criterio no lineal, puramente empírico, que permite valorar la rotura de un medio rocoso mediante la introducción de las principales características geológicas y geotécnicas.

La versión original del criterio de rotura de HoekBrown fue introducida en el 1980 desde entonces su uso se ha generalizado en el ámbito de la mecánica de rocas, el criterio ha sufrido varias modificaciones, así como la introducción de nuevos parámetros para definir el estado del material, y nuevas propuestas para obtener la caracterización del macizo, la última en 2002. (Ros Avila, 2008).

Read \& Stacey (2019) explican la importancia de la orientación de las discontinuidades subparalelas al talud en el análisis de estabilidad de taludes de la siguiente manera:

Los criterios de resistencia como el criterio de Hoek-Brown suponen que el macizo rocoso está formado por un grupo isotrópico de piezas de roca intactas, con discontinuidades estrechamente separadas para las que no existe una dirección de falla preferida, lo que raramente es el caso. Es posible definir la fuerza de cizallamiento direccional para el macizo rocoso tal como sigue.

a. Definir la resistencia a la rotura básica o isotrópica del macizo rocoso mediante el criterio Hoek-Brown Generalizado y definir valores equivalentes para la cohesión y el ángulo de fricción de la roca.

b. Si no hay conjuntos de discontinuidad (fallas y/o juntas) paralelos a la pendiente, se puede suponer que la resistencia al corte del macizo rocoso es isotrópica y corresponde a la resistencia básica definida en (a).

c. Si hay uno o más conjuntos de discontinuidad paralelos a la pendiente, la resistencia al corte del macizo rocoso no puede ser asumida isotrópico, porque el macizo rocoso es más débil en la dirección de estas discontinuidades. Por lo tanto, la resistencia a la rotura del macizo rocoso es mucho menor en la dirección de estas discontinuidades. En la dirección normal a las discontinuidades, la resistencia al cizallamiento será igual a la resistencia de masa de roca básica definida en (a) y en la dirección paralela a las discontinuidades será igual a la resistencia al corte de las discontinuidades.

Edelbro (2004) en el desarrollo de la tesis de licenciatura "Evaluation of Rock Mass Strength Criteria" indica que, la mayoría de los softwares desarrollados para el análisis de mecánica de suelos y rocas proporciona el modelo constitutivo de Mohr- Coulomb para la simulación del comportamiento plástico. Sin embargo, no existe un método directo generalmente aceptado para estimar los parámetros de Mohr- Coulomb (cohesión y ángulo de fricción) de un macizo rocoso. Por lo antes mencionado, se vuelve interesante evaluar los parámetros utilizando un análisis de regresión lineal de la envolvente de falla no lineal de Hoek y Brown, lo cual permitiría utilizar el criterio de Hoek-Brown como entrada para el análisis numérico.

En el desarrollo del presente trabajo de investigación el uso del criterio de Mohr- Coulomb se limita a las discontinuidades, mas no al macizo rocoso, para el cual se utilizó el criterio de Hoek-Brown Generalizado.

Lo inédito de la investigación es demostrar que a medida que la calidad del macizo rocoso aumenta mayor es el porcentaje de variación del factor de seguridad en el macizo rocoso anisotrópico respecto al isotrópico.

\section{MATERIALES Y MÉTODOS}

En la Figura 1 se esquematiza las diferentes etapas de trabajo y se describe brevemente las metodologías empleadas en cada una de ellas.

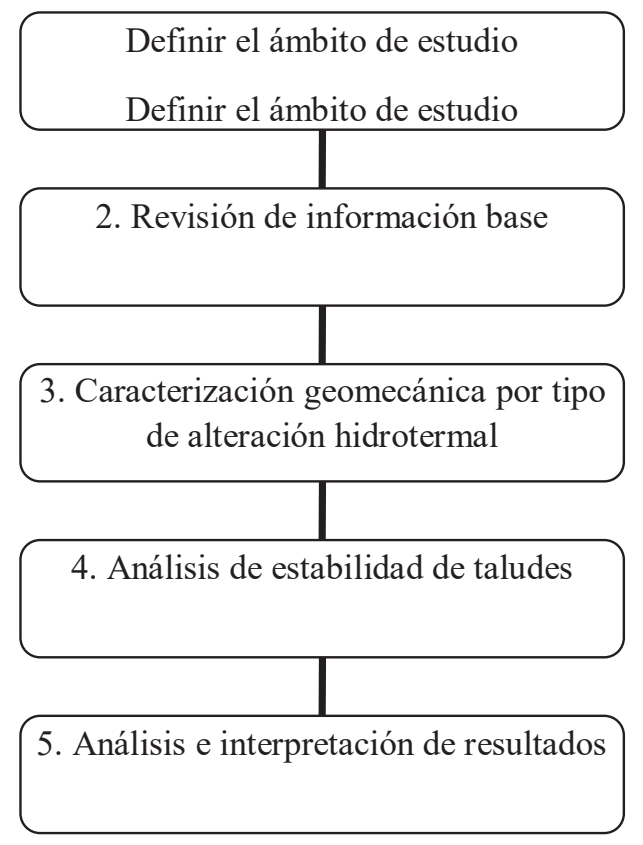

Figura 1. Esquema de trabajo

Los métodos empleados son combinaciones los pasos mencionados en la Figura 1. En la primera etapa comprende la selección del ámbito de estudio de acuerdo con la evaluación de la información y búsqueda de trabajos similares a nivel internacional y a nivel nacional. La segunda etapa consistió en la revisión estudios geológicos - geotécnicos, hechos en la zona de estudio, revisando principalmente las alteraciones hidrotermales presentes en el yacimiento Jésica. En la tercera etapa se realizó la caracterización geomecánica por tipo de alteración hidrotermal en once (11) taladros diamantinos y ochenta (80) estaciones geomecánicas del tajo abierto Jésica. La cuarta etapa corresponde al análisis de estabilidad de taludes en el software Slide versión 6.0 para determinar los factores de seguridad. La quinta etapa comprendió el 
análisis y la interpretación de resultados; determinado que el factor de seguridad de un macizo rocoso anisotrópico es menor al factor de seguridad anisotrópico.

La investigación realizada es de enfoque cuantitativo, diseño no experimental, transeccional de alcance descriptivo - correlacional.

\section{RESULTADOS}

\subsection{Alteraciones hidrotermales}

Las alteraciones hidrotermales presentes en el tajo abierto Jésica, son Clay Pirita (CP) y Sílice Clay Pirita (SCP), presentes en la zona de sulfuros; las alteraciones Sílice Clay (SC), Sílice Alunita (SA), Sílice Granular (SG) y Sílice Masiva (SM) están presentes en la zona de óxidos y sulfuros. La alteración SA es predominante en dirección noreste a suroeste del tajo abierto Jésica, mientras que la alteración SCP predomina en el sector oeste, la alteración SG se ubica en la zona central junto con la SM, las alteraciones $\mathrm{SC}$ y $\mathrm{CP}$ se ubican principalmente en las zonas periféricas del tajo.

\subsection{Caracterización y Clasificación del Macizo Rocoso}

El método de clasificación Rock Mass Rating (RMR), fue desarrollado por Bieniawski en 1973, con actualizaciones en 1979 y 1989, González de Vallejo et al., p.230 (2002).

González de Vallejo et al., (2002) indican que el parámetro que define la clasificación es el denominado índice RMR, que indica la calidad del macizo rocoso en cada dominio estructural a partir de los siguientes parámetros:
- Resistencia a la compresión simple de la matriz rocosa (UCS).

- Grado de fracturación del macizo rocoso (RQD).

- Espaciado de las discontinuidades o frecuencia de fracturas por metro (FF).

- Condiciones de las discontinuidades (JC), el cual consiste en considerar los siguientes parámetros:

- Abertura de las caras de la discontinuidad.

- Continuidad o persistencia de la discontinuidad.

- Rugosidad.

- $\quad$ Alteración de la discontinuidad.

- Relleno de las discontinuidades.

- Presencia del agua (WC).

Para obtener el índice RMR de Bieniawski se suma las cinco (5) variables o parámetros calculados, eso da como resultado un valor índice (RMR básico).

Se realizó la caracterización geomecánica de acuerdo RMR de Bieniawski 1989. En las Tablas 1 y 2, se muestra el resumen de parámetros para cada tipo de alteración en la zona de óxidos y sulfuros, observando que para una misma alteración en la zona de sulfuros mejoran el RMR del macizo rocoso, respecto a la zona de óxidos.

El valor UCS se tomó de ensayos a la compresión simple, el RQD, la frecuencia de fracturas (FF), la condición de las discontinuidades (JC) y la condición de

Tabla 1. Resumen de los parámetros RMR89, por tipo de alteración en óxidos

\begin{tabular}{|c|c|c|c|c|c|c|c|}
\hline \multicolumn{2}{|c|}{ Alteración } & \multicolumn{5}{|c|}{ Parámetros RMR 89} & \multirow{2}{*}{ RMR } \\
\hline Descripción & Símbolo & UCS (MPa) & $\mathrm{RQD}(\%)$ & FF & $\mathrm{JC}$ & WC & \\
\hline Sílice Clay & SC & 63.67 & 40.76 & 11.46 & 11.43 & 15.00 & 47 \\
\hline Sílice Granular & SG & 60.43 & 38.93 & 13.52 & 13.00 & 15.00 & 49 \\
\hline Sílice Masiva & SM & 73.42 & 42.78 & 13.18 & 13.72 & 15.00 & 52 \\
\hline Sílice Alunita & SA & 54.55 & 60.75 & 8.39 & 14.08 & 15.00 & 56 \\
\hline
\end{tabular}

Fuente: Elaboración propia

Tabla 2. Resumen de los parámetros RMR89, por tipo de alteración en sulfuros

\begin{tabular}{|c|c|c|c|c|c|c|c|}
\hline \multicolumn{2}{|c|}{ Alteración } & \multicolumn{5}{|c|}{ Parámetros RMR 89} & \multirow{2}{*}{ RMR } \\
\hline Descripción & Símbolo & UCS (MPa) & RQD (\%) & FF & $\mathrm{JC}$ & WC & \\
\hline Clay Pirita & $\mathrm{CP}$ & 18.50 & 34.96 & 15.25 & 9.16 & 15.00 & 40 \\
\hline Sílice Clay Pirita & SCP & 38.26 & 37.25 & 10.70 & 10.60 & 15.00 & 45 \\
\hline Sílice Clay & SC & 42.94 & 58.42 & 8.42 & 13.79 & 15.00 & 54 \\
\hline Sílice Granular & $S G$ & 59.72 & 61.61 & 7.44 & 16.06 & 15.00 & 59 \\
\hline Sílice Alunita & SA & 65.85 & 68.91 & 5.47 & 15.08 & 15.00 & 60 \\
\hline Sílice Masiva & SM & 99.49 & 62.99 & 6.49 & 16.31 & 15.00 & 61 \\
\hline
\end{tabular}

Fuente: Elaboración propia 
agua (WC) son parámetros registrados durante el logueo geomecánico. Finalmente, a cada parámetro le corresponde un valor de acuerdo con la clasificación RMR, obteniendo como resultado final el índice RMR para cada tipo de alteración hidrotermal.

En el tajo abierto Jésica existe cuatro (4) tipos de alteración hidrotermal en la zona de óxidos (SA, SM, SG y SC) y seis (6) tipos de alteración hidrotermal en la zona de sulfuros (SA, SM, SG, SC, SCP y CP). Cada tipo de alteración hidrotermal presenta valores de caracterización geomecánica (GSI) distintos, en óxidos SC (42), SG (44), SM (47), SA (51), en sulfuros los valores son CP (35), SCP (40), SC (49), SG (54), SA (55), SM (56). Se observa que en la zona de sulfuros los valores de GSI son mayores que en la zona de óxidos para las mismas alteraciones.

\subsection{Propiedades geomecánicas del macizo rocoso}

Las propiedades geomecánicas del macizo rocoso, se muestran en las Tablas 3 y 4 , en óxidos y sulfuros respectivamente.

Tabla 3. Propiedades geomecánicas del macizo rocoso en óxidos

\begin{tabular}{ccccc}
\hline $\begin{array}{c}\text { Alteración } \\
\text { Símbolo }\end{array}$ & UCS & GSI & mi & D \\
\hline SC & 63.67 & 42 & 12 & 0.9 \\
SG & 60.43 & 44 & 18 & 0.9 \\
SM & 73.42 & 47 & 18 & 0.9 \\
SA & 54.55 & 51 & 20 & 0.9 \\
\hline
\end{tabular}

Fuente: Elaboración propia

Tabla 4. Propiedades geomecánicas del macizo rocoso en sulfuros

\begin{tabular}{ccccc}
\hline $\begin{array}{c}\text { Alteración } \\
\text { Símbolo }\end{array}$ & UCS & GSI & mi & D \\
\hline CP & 18.50 & 35 & 10 & 0.9 \\
SCP & 38.26 & 40 & 12 & 0.9 \\
SC & 42.94 & 49 & 20 & 0.9 \\
SG & 59.72 & 54 & 20 & 0.9 \\
SA & 65.85 & 55 & 21 & 0.9 \\
SM & 99.49 & 56 & 21 & 0.9 \\
\hline
\end{tabular}

Fuente: Elaboración propia

El parámetro Geological Strength Index (GSI) es utilizado para la estimación de los parámetros de entrada para el cálculo de la resistencia, el cual se obtuvo a partir del RMR, aplicando los criterios siguientes RMR89 $>23 \rightarrow$ $\mathrm{GSI}=\mathrm{RMR} 89-5$

RMR89 $<23 \rightarrow$ No se puede utilizar el RMR89 para la obtención del GSI.

El parámetro UCS, se obtuvo de la caracterización del macizo rocoso y la constante mi, está en función de las propiedades geomecánicas del macizo rocoso.

D es denominado factor de disturbancia, "es un factor que depende del grado de alteración al que ha sido sometido el macizo rocoso por los efectos de las voladuras o por la relajación de esfuerzos" Hoek et al., p.2 (2002). En un tajo abierto, el factor D, varía desde 0.7 hasta 1.0 (Hoek et al., 2002) para el caso de la investigación se consideró un valor de 0.9 .

Los parámetros de las Tablas 3 y 4 fueron los datos de entrada para el criterio de Hoek- Brown Generalizado y Anisotrópico Generalizado en el software Slide 6.0.

Asimismo, para el criterio Anisotrópico Generalizado se requiere los parámetros del criterio de rotura de Mohr - Coulomb. Estos parámetros se obtuvieron a partir de ensayos de corte directo, los resultados fueron cohesión 0.0 $\mathrm{kN} / \mathrm{m}^{2} \mathrm{y}$ ángulo de fricción $30^{\circ}$.

\subsection{Análisis de estabilidad de taludes del macizo rocoso isotrópico y anisotrópico}

Con los datos de las Tablas 3 y 4 se realizó el análisis de estabilidad considerando el talud con altura de $300 \mathrm{~m}$ y un ángulo de $45^{\circ}$. El análisis de estabilidad se realizó en el software Slide versión 6.0, considerando el macizo rocoso isotrópico (falla circular) aplicando el criterio de Hoek y Brown Generalizado y el macizo rocoso anisotrópico (falla no circular) aplicando el criterio de rotura Anisotrópico Generalizado.

Para el análisis de estabilidad física del macizo rocoso anisotrópico se consideraron dos (2) familias de discontinuidades sub paralelas al talud, denominadas $\mathrm{F} 1$ (buzamiento de $72^{\circ}$ a $82^{\circ}$ ) y F2 (buzamiento de $45^{\circ}$ a $55^{\circ}$ ) predominantes en el tajo abierto Jésica.

En las Tablas 5 y 6 se muestra los resultados del análisis de estabilidad de taludes para cada tipo de alteración hidrotermal en la zona de óxidos y sulfuros respectivamente.

En ambos casos se observa los factores de seguridad obtenidos considerando el macizo rocoso isotrópico y anisotrópico, así como la variación del factor de seguridad de acuerdo con la orientación de la discontinuidad F1 (buzamiento de $72^{\circ}$ a $82^{\circ}$ ) y F2 (buzamiento de $45^{\circ}$ a $55^{\circ}$ ).

Los resultados indican que el factor de seguridad disminuye cuando se realiza el análisis de estabilidad considerando el macizo rocoso anisotrópico, es decir las discontinuidades subparalelas al talud, así también mientras mayor es la calidad del macizo rocoso mayor es la variación (disminución) del factor de seguridad anisotrópico respecto al isotrópico.

La Figura 2, muestra la correlación entre el factor de seguridad del macizo rocoso isotrópico y el factor de seguridad del macizo rocoso anisotrópico en la zona de óxidos, representada por una ecuación lineal y siendo el valor $\mathrm{R}^{2}$ de 1.0 para las cuatro (4) alteraciones hidrotermales, explicando el $100 \%$ de los datos.

La Figura 3, muestra la correlación entre el factor de seguridad del macizo rocoso isotrópico y el factor de seguridad del macizo rocoso anisotrópico en la zona de sulfuros, representada por una ecuación lineal y siendo el valor $\mathrm{R}^{2}$ de 1.0 para las seis (6) alteraciones hidrotermales, explicando el $100 \%$ de los datos. 
Tabla 5. Resultados del análisis de estabilidad isotrópico y anisotrópico en óxidos

\begin{tabular}{cccccccc}
\hline $\begin{array}{c}\text { Alteración } \\
\text { Símbolo }\end{array}$ & GSI & RMR & Isotrópico & Factor de seguridad & \multicolumn{3}{c}{ Variación del factor de seguridad } \\
Anisotrópico & F1 & F2 & F1 & F2 \\
\hline SC & 42 & 47 & 1.45 & 1.38 & 1.21 & $-5 \%$ & $-16 \%$ \\
SG & 44 & 49 & 1.61 & 1.55 & 1.31 & $-4 \%$ & $-19 \%$ \\
SM & 47 & 52 & 1.81 & 1.70 & 1.40 & $-6 \%$ & $-23 \%$ \\
SA & 51 & 56 & 1.91 & 1.80 & 1.46 & $-6 \%$ & $-24 \%$ \\
\hline
\end{tabular}

Fuente: Elaboración propia

Tabla 6. Resultados del análisis de estabilidad isotrópico y anisotrópico en sulfuros

\begin{tabular}{cccccccc}
\hline $\begin{array}{c}\text { Alteración } \\
\text { Símbolo }\end{array}$ & GSI & RMR & Isotrópico & Factor de seguridad & \multicolumn{3}{c}{ Variación del factor de seguridad } \\
Anisotrópico & F1 & F2 & F1 & Anisotrópico \\
\hline CP & 35 & 40 & 0.81 & 0.80 & 0.75 & $-1 \%$ & $-8 \%$ \\
SCP & 40 & 45 & 1.16 & 1.12 & 1.03 & $-4 \%$ & $-12 \%$ \\
SC & 49 & 54 & 1.75 & 1.66 & 1.37 & $-5 \%$ & $-22 \%$ \\
SG & 54 & 59 & 2.07 & 1.93 & 1.53 & $-7 \%$ & $-26 \%$ \\
SA & 55 & 60 & 2.25 & 2.09 & 1.66 & $-7 \%$ & $-26 \%$ \\
SM & 56 & 61 & 2.56 & 2.34 & 1.81 & $-8 \%$ & $-29 \%$ \\
\hline
\end{tabular}

Fuente: Elaboración propia

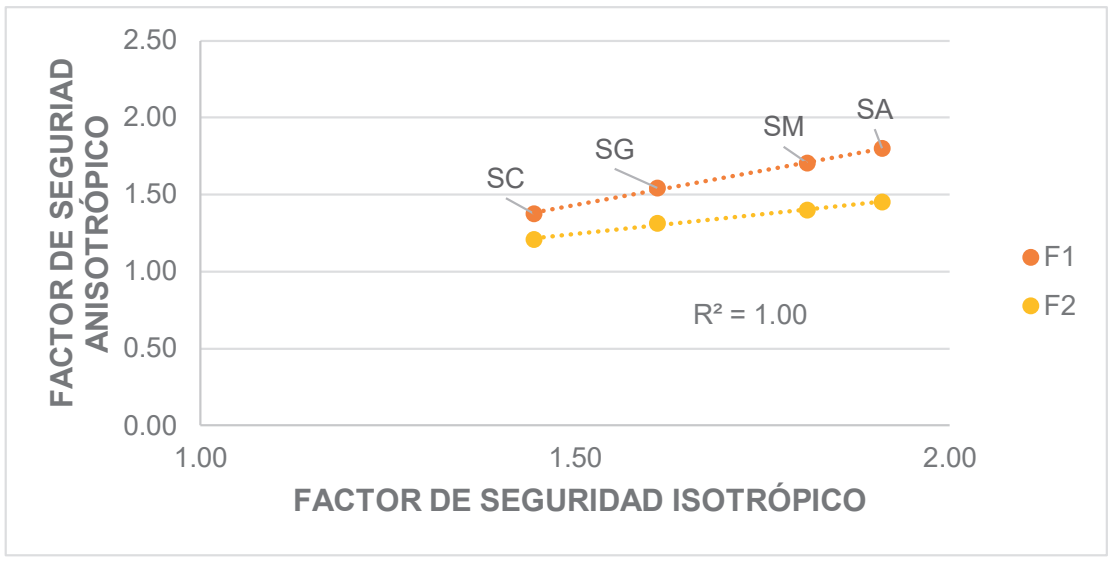

Figura 2. Correlación y ecuación lineal para los factores de seguridad obtenidos en el macizo rocoso isotrópico y anisotrópico - zona de óxidos. Fuente: Elaboración propia.

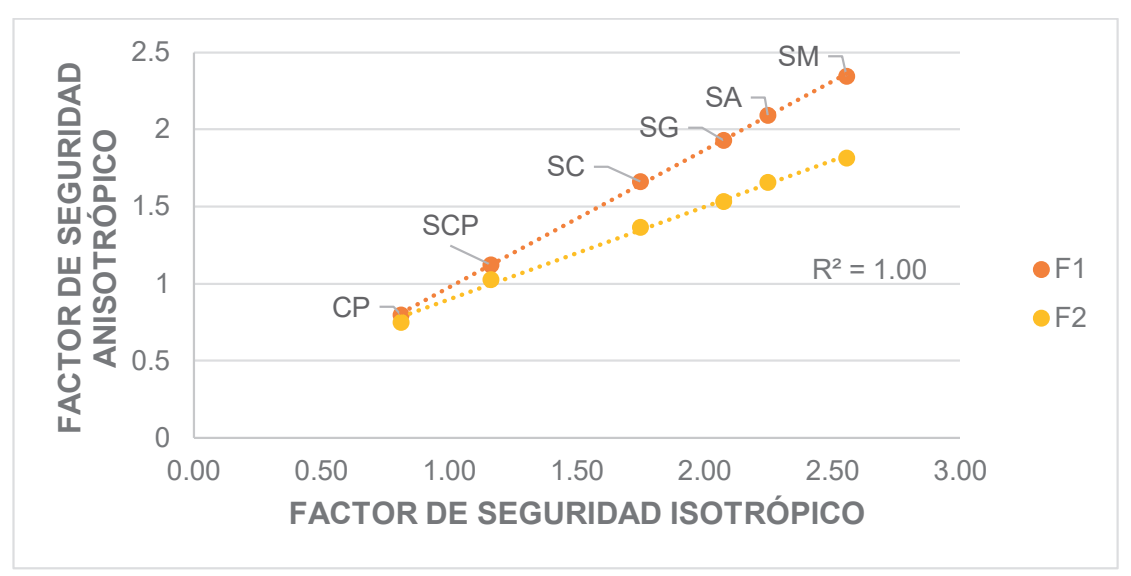

Figura 3. Correlación y ecuación lineal para los factores de seguridad obtenidos en el macizo rocoso isotrópico y anisotrópico - zona de sulfuros. Fuente: Elaboración propia. 
La Figura 4, muestra la variación del factor de seguridad del macizo rocoso anisotrópico respecto al macizo rocoso isotrópico en la zona de óxidos, donde se observa que la variación es mayor cuando se considera la orientación de la discontinuidad F2 (buzamiento de $45^{\circ}$ a $55^{\circ}$ ), respecto a la orientación de la discontinuidad $\mathrm{F} 1$ (buzamiento de $72^{\circ}$ a $82^{\circ}$ ), es decir cuando el buzamiento es más cercano al buzamiento del talud analizado de $45^{\circ}$ mayor es la disminución del factor de seguridad.

En la Figura 5, se muestra de variación del factor de seguridad del macizo rocoso anisotrópico respecto al macizo rocoso isotrópico en la zona de sulfuros, donde se aprecia la misma tendencia que en la zona de óxidos, la discontinuidad más desfavorable es la F2.

\section{DISCUSIÓN}

Se realizó el análisis de estabilidad por tipo de alteración hidrotermal en la zona de óxidos y sulfuros del tajo abierto Jésica, considerando el macizo rocoso isotrópico y anisotrópico.

Para el macizo rocoso anisotrópico se consideraron dos (2) familias de discontinuidades subparalelas al talud,
F1 (buzamiento de $72^{\circ}$ a $82^{\circ}$ ) y F2 (buzamiento de $45^{\circ}$ a $55^{\circ}$ ), en ambos casos el factor de seguridad anisotrópico es menor al factor de seguridad isotrópico; con la familia F1 el factor de seguridad disminuye entre 5 a $6 \%$ en la zona de óxidos y de 1 a $8 \%$ en la zona de sulfuros, con la familia F2 el factor de seguridad disminuye de 16 a $24 \%$ en la zona de óxidos y de $8 \%$ a $29 \%$ en la zona de sulfuros.

Los resultados obtenidos para los factores de seguridad isotrópicos y anisotrópicos son concordantes con lo mencionado por Karzulovic \& Read (2009), debido a que los factores de seguridad del macizo rocoso anisotrópico son menores a los factores de seguridad del macizo rocoso isotrópico, debido a la presencia de discontinuidades subparalelas al talud analizado.

Así también es importante recalcar que a medida que la calidad del macizo rocoso (RMR) aumenta, mayor es la disminución del factor de seguridad anisotrópico respecto al isotrópico; esto a su vez refleja que a mayor diferencia de propiedades de resistencia entre el macizo rocoso y las discontinuidades paralelas o subparalelas al talud, mayor es la variación del factor de seguridad.

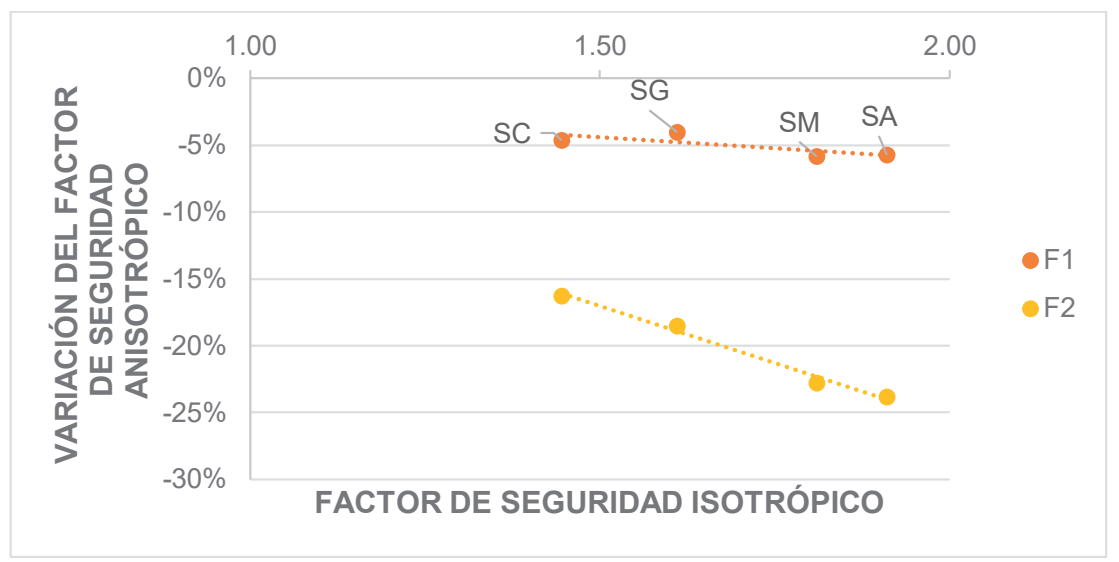

Figura 4. Variación de los factores de seguridad obtenidos en el macizo rocoso anisotrópico respecto al isotrópico - zona de óxidos. Fuente: Elaboración propia.

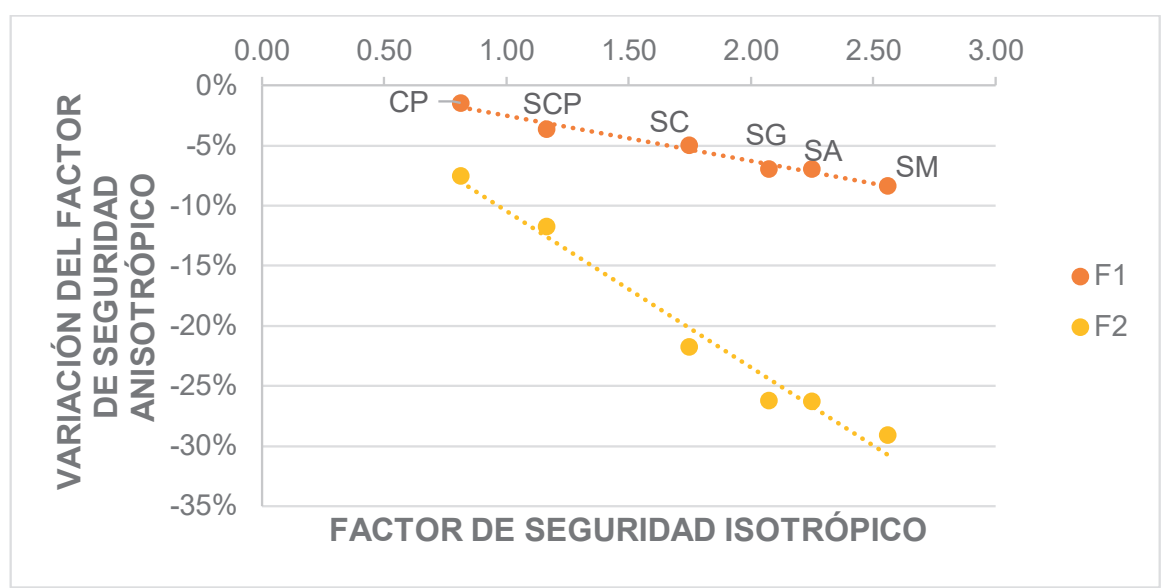

Figura 5. Variación de los factores de seguridad obtenidos en el macizo rocoso anisotrópico respecto al isotrópico - zona de sulfuros. Fuente: Elaboración propia. 


\section{CONCLUSIONES}

1. Cada alteración hidrotermal se asocia con un determinado valor de RMR; altos valores de RMR, se asocian con altos valores de factor de seguridad. En la zona de óxidos los valores de RMR varían de 47 a 56 y los factores de seguridad isotrópicos de 1.45 a 1.91 , y en la zona de sulfuros los valores de RMR varían de 40 a 61 y los factores de seguridad isotrópicos de 0.81 a 2.34 .

2. Se ha determinado que el factor de seguridad de los taludes disminuye si se realiza un análisis de estabilidad anisotrópico, disminuyendo de 5\% a $24 \%$ en óxidos y de $1 \%$ a $29 \%$ en sulfuros.

3. A mayor calidad del macizo rocoso (RMR), mayor es la disminución del factor de seguridad anisotrópico respecto al factor de seguridad isotrópico, esta diferencia es más marcada en la zona de sulfuros con la familia F2, donde en un macizo rocoso con RMR 40 el factor de seguridad disminuye en $8 \%$, mientras que en un macizo rocoso con RMR 61 el factor de seguridad disminuye en $29 \%$.

\section{AGRADECIMIENTOS}

A la unidad de posgrado de la facultad de Ingeniería Geológica, Minera, Metalúrgica y Geográfica de la Universidad Nacional Mayor de San Marcos, y al Instituto de Investigación de la FIGMMG por facilitar la publicación del presente artículo.

\section{REFERENCIAS}

Edelbro, C. (2004). Evaluation of rock mass strength criteria. Luleå University of Technology, 98. https://www.diva-portal. org $/$ smash $/$ record.jsf?pid=diva2\%3A990018\&dswid=4457

González de Vallejo, L. I. ., Ferrer, M., Ortuño, L., \& Oteo, C. (2002). Ingeniería Geológica. Madrid: Pearson Educación. http://www.ingebook.com/ib/NPcd/IB_BooksVis?cod_ primaria $=1000187 \&$ codigo_libro $=1237$

Hoek, E., Carranza-Torres, C., Corkum, B., Hoek, E., \& CarranzaTorres, C. (2002). Hoek-Brown failure criterion-2002 Edition (Vol. 1). www.rocscience.com.

Read, J., \& Stacey, P. (2019). Guidelines for Open Pit Slope Design. In Guidelines for Open Pit Slope Design. https:// doi.org/10.1071/9780643101104

Ros Avila, J. (2008). Análisis comparativo de los criterios de rotura de Hoek\&Brown y Mohr-Coulomb en el estudio de estabilidad en macizos rocosos. Minor Thesis. https:// upcommons.upc.edu/handle/2099.1/6529?localeattribute $=$ en 
